\title{
The effect of the parent graphite on the structure of graphene oxide
}

Cristina Botas ${ }^{1}$, Patricia Álvarez ${ }^{1}$, Clara Blanco ${ }^{1}$, Ricardo Santamaría ${ }^{1}$, Marcos

Granda $^{1}$, Pablo Ares ${ }^{2}$, Francisco Rodríguez-Reinoso ${ }^{3}$ and Rosa Menéndez ${ }^{1 *}$

${ }^{1}$ Instituto Nacional del Carbón (INCAR-CSIC), Apdo. 73, 33080, Oviedo (Spain)

${ }^{2}$ Nanotec Electrónica S.L., Apdo. 12, 28760 Tres Cantos, Madrid (Spain)

${ }^{3}$ Dept. Química Inorgánica, Universidad de Alicante, Apdo 99, E-03080 Alicante (Spain)

\section{ABSTRACT}

The effect of the parent graphite on the structure of graphene oxide (GO) is investigated using high purity graphites with a uniform crystallite size. Our results provide direct evidence of how the size of the graphite crystal affects the oxidation process and the functionality and sheet size of the resulting GO. The important role of the crystal boundaries in the graphite with smaller crystallites is confirmed by the smaller size of the GO sheets obtained and also by the presence of carboxylic groups, located at the edges of the sheets. However, functionalization in the graphite with larger crystals mainly occurs in the vicinity of basal plane defects, as evidenced by the larger number of epoxy groups. Thus, this study leads to a better understanding of the oxidation process of graphite and provides a way to produce GOs suitable for different applications.

\footnotetext{
* Correspondig author: Fax. + 349852976 62; E-mail: rosmenen@incar.csic.es (Prof. R. Menéndez)
} 


\section{Introduction}

A great deal of effort has been devoted in recent years to the synthesis of graphene by different methods [1-5] and especially to the study of its electronic behavior [6] in the search for potential applications. The preparation of graphene by chemical methods such as the graphene oxide route[2] offers the possibility of producing it on a large scale and, at the same time, of controlling its quality, depending on the properties of: i) the parent graphite, ii) the oxidation method used, and iii) the final reduction of the GO to graphene. In this regard, GO and partially reduced graphene oxides are graphene derivatives that exhibit a structure decorated with defects produced during the oxidation and/or reduction processes. However, the complexity of the reactions involved in the overall process (oxidation, exfoliation, reduction), in addition to the numerous variables that may affect the bonding of the carbon atoms of the parent graphite, makes strict control of the yield and quality of the products very difficult. This is a handicap for the understanding of the mechanisms involved and their subsequent effect on the final product. It is generally accepted that the continuous aromatic lattice of graphene in GO is interrupted by oxygen surface groups (mainly epoxy, hydroxyl and carbonyl groups), the carboxylic groups being located at the edges [7]. However, it is difficult to determine the exact structure of GO. A very important factor that must be taken into account is the contribution of the characteristics of the parent graphite, but there is very little detailed information available on this $[8,9]$. The main reason for this lack of information is that the properties measured in any given graphite represent the global properties of the constituents as a whole, which is a blend of crystalline structures of various sizes and orientations. Even highly oriented pyrolytic graphite (HOPG), which has frequently been used in the synthesis of GO, is not composed of just a single crystal 
but consists of grains with different orientations [10]. Previous studies on the behavior of graphite during oxidation to produce graphite oxide have shown that texture and crystal size play an important role in the mechanisms involved, indicating that preferential attack is directed at the edges of the graphite particle at the single crystal boundaries and at the defects present in the basal planes [11].

In order to tackle the questions that still remain unanswered, we proposed a study consisting of the following steps: i) the use of a common organic precursor (synthetic coal-based pitch consisting of a mixture of polycyclic aromatic hydrocarbons) for the production of ash-free graphitic materials (inorganic material is always present in natural graphite) of varying crystal size; and ii) the synthesis and characterization of GO from these graphites.

\section{Experimental}

\subsection{Preparation of graphites.}

Two cokes of different optical texture (mosaics and flow domains) obtained from two synthetic pitches prepared from the same coal fraction at different conditions [12] were ground in an agate ball mill at $400 \mathrm{rpm}$ for $1 \mathrm{~h}$, and then sieved through a $0.75 \mu \mathrm{m}$ mesh. The resultant powders from each coke were graphitized at $2800{ }^{\circ} \mathrm{C}$ in a graphite furnace for 45 min under argon flow to provide two graphites, G1 and G2. The heating rates selected were: i) $50{ }^{\circ} \mathrm{C} \min ^{-1}$ from room temperature to $700{ }^{\circ} \mathrm{C}$, ii) $100{ }^{\circ} \mathrm{C} \min ^{-1}$ from $700{ }^{\circ} \mathrm{C}$ to $1000{ }^{\circ} \mathrm{C}$, iii) $20{ }^{\circ} \mathrm{C} \min ^{-1}$ in the $1000-2000{ }^{\circ} \mathrm{C}$ interval and iv) $10{ }^{\circ} \mathrm{C}$ $\min ^{-1}$ from $2000{ }^{\circ} \mathrm{C}$ to $2800{ }^{\circ} \mathrm{C}$.

\subsection{Preparation of GO.}


The GOs were synthesized from the synthetic graphites by the modified Hummers method [13]. This method makes use of the Hummers reagents with additional amounts of $\mathrm{NaNO}_{3}$ and $\mathrm{KMnO}_{4}$. Concentrated $\mathrm{H}_{2} \mathrm{SO}_{4}(360 \mathrm{~mL})$ was added to a mixture of synthetic graphite $(7.5 \mathrm{~g})$ and $\mathrm{NaNO}_{3}(7.5 \mathrm{~g})$, and the mixture was cooled down to $0{ }^{\circ} \mathrm{C}$ using an ice bath. $\mathrm{KMnO}_{4}(45 \mathrm{~g})$ was added slowly in small doses to keep the reaction temperature below $20{ }^{\circ} \mathrm{C}$. The solution was heated to $35{ }^{\circ} \mathrm{C}$ and stirred for $3 \mathrm{~h}$, at which point $3 \%$ of $\mathrm{H}_{2} \mathrm{O}_{2}(1.5 \mathrm{~L})$ was added slowly, giving rise to a pronounced exothermal effect up to $98^{\circ} \mathrm{C}$. The reaction mixture was stirred for $30 \mathrm{~min}$ and, finally, the mixture was centrifuged (3700 rpm for $30 \mathrm{~min}$ ), the supernatant being decanted away. The remaining solid material was then washed with $600 \mathrm{~mL}$ of water and centrifuged again, this process being repeated until the $\mathrm{pH}$ was neutral. A colloidal suspension of individual $\mathrm{GO}$ sheets in purified water $\left(1 \mathrm{mg} \mathrm{mL}^{-1}\right)$ was prepared in 1 - $\mathrm{L}$ batches, and kept under ultrasound for $10 \mathrm{~h}$. Then the suspension was centrifuged $(3700 \mathrm{rpm}$ for 30 min), the supernatant was filtered over cellulose and the solid was discarded. To prepare the suspensions in organic solvents the water was evaporated in a rotary evaporator and $10 \mathrm{~mL}$ of each solvent was added to $1 \mathrm{mg}$ of solid, after which the mixture was sonicated for $30 \mathrm{~min}$.

\subsection{Characterization of graphites}

The optical texture of the graphites was determined by polarized light microscopy using a Zeiss Axioplan microscopy, equipped with an adjusted ocular (10X), an oil-immersion objective (20X, 50X and 100X), a polarizer and a one-wave retarder plate to generate interference colours. Prior to the analysis, the samples were embedded in an epoxy resin, then polished and finally examined using polarized light. The X-ray diffraction of graphites was performed using a Bruker D8 Advance diffractometer. The radiation 
frequency used was the $\mathrm{K} \alpha 1$ line from $\mathrm{Cu}(1.5406 \AA)$, with a power supply $40 \mathrm{KV}$ and $40 \mathrm{~mA}$. The crystallite size along the c-axis (Lc) was obtained from the (002) reflection of the XRD patterns, which were recorded at steps of $0.01^{\circ}$ and intervals of $6 \mathrm{~s}$ per step, using the Scherrer equation. The crystallite size along the a-axis (La) was calculated from the (100) Bragg peak. The carbon content of the samples was determined by elemental analysis with a LECO-CHNS-932 microanalyzer. The analyses were performed using $1 \mathrm{mg}$ of ground sample. The results were quoted from an average of the values of four determinations. In all cases, the experimental error was $<0.5 \%$ of the absolute value. The ash content was determined by TGA on a TA SDT 2960 analyzer

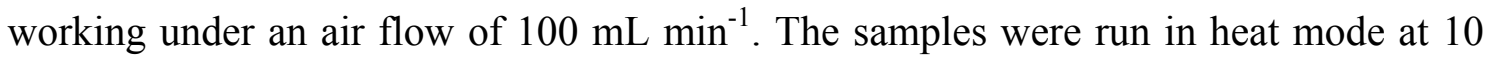
${ }^{\circ} \mathrm{C} \min ^{-1}$ from $40{ }^{\circ} \mathrm{C}$ to $1000{ }^{\circ} \mathrm{C}$. Particle size analysis measurements were carried out using a laser scattering particle size distribution analyzer (COULTER BEKMAN LD 13 320) and methanol as solvent.

\subsection{Characterization of $G O$}

XPS measurements of GOs were carried out on a SPECS system operating under $10^{-7}$ $\mathrm{Pa}$ connected to a $\mathrm{Mg} \mathrm{Ka} \mathrm{X}$-ray source $(100 \mathrm{~W})$. All of the spectra were energy calibrated by assigning $284.5 \mathrm{eV}$ to the $\mathrm{C} 1 \mathrm{~s}$ binding energy of the 'graphitic' peak. Relative atomic concentrations were calculated by integrating the peak areas according to the Shirley background correction procedure and using atomic sensitivity factors provided elsewhere [14]. To evaluate the functional groups of the samples, the XPS C1s peaks were curve-fitted using a Gaussian-Lorentzian peak shape [15]. FTIR spectra of GOs were recorded at room temperature using an attenuated total reflection (ATR) mode with a diamond plate of one bounce and a Fourier transform infrared spectrometer (FTIR, Nicolet 8700 FTIR, Thermo Scientific) fitted with a DFT (deuterated triglycine 
sulfate) detector. Solid samples were placed on the germanium plate. 64 scans $\left(4 \mathrm{~cm}^{-1}\right.$ resolution) per sample were recorded between 3700 and $600 \mathrm{~cm}^{-1}$. Raman spectra were recorded from750 to $3500 \mathrm{~cm}^{-1}$ on a Renishaw 2000 Confocal Raman Microprobe (Rhenishaw Instruments, England) using a 514.5-nm argon ion laser. The variation in the weight of the sample during thermal treatment was monitored by TGA using a TA SDT 2960 analyzer. $5 \mathrm{mg}$ of each sample was placed in a crucible which was then introduced into the thermobalance. The temperature was increased to $1000{ }^{\circ} \mathrm{C}$ at a heating rate of $10{ }^{\circ} \mathrm{C} \mathrm{min}^{-1}$ under a nitrogen flow of $100 \mathrm{~mL} \mathrm{~min}$.

TEM observations were performed on a JEOL 2000 EX-II instrument operated at 160 $\mathrm{kV}$. AFM characterizations of the GO sheets were carried out using a Cervantes atomic force microscope from Nanotec Electronica ${ }^{\mathrm{TM}}$ operating under ambient conditions. The oxidized material from the suspensions was deposited on mica substrates by drop casting. The solution was left for ten minutes on the mica, then washed with purified water and dried with nitrogen. Samples were imaged under exactly the same conditions $\left(\approx 20{ }^{\circ} \mathrm{C}\right.$ temperature and $\approx 30 \%$ relative humidity). Nanosensor ${ }^{\mathrm{TM}} \mathrm{PPP}-\mathrm{NCH}$ PointProbe $^{\circledR}$ Plus microcantilevers were used to image the GO sheets via attractive regime amplitude-modulated mode imaging [16], thus to ensure that the sheets would not be deformed by the microcantilever tip. The GO sheet heights and areas were measured using more than 75 different sheets in each sample. In order to obtain a more statistical result for the sheet height values, histograms were constructed by measuring the distance between the first peak, corresponding to the substrate, and the second peak, corresponding to each sheet height (Figure 1a). To obtain the area values, the Flooding option from WSxM software was used (Figure 1b). This option directly gives the number of sheets in an image, the area of each individual sheet, the total area of the sheets and the area histogram. The Flooding option allows the highest points of an 
image to be detected. All points in an image lower than a configurable minimum height will be "flooded", to give the island distributions and all the related statistical information. There is also a minimum area value for discriminating spurious points on the image. For more details on the Flooding option the WSxM help menu can be consulted (Figure 1b).
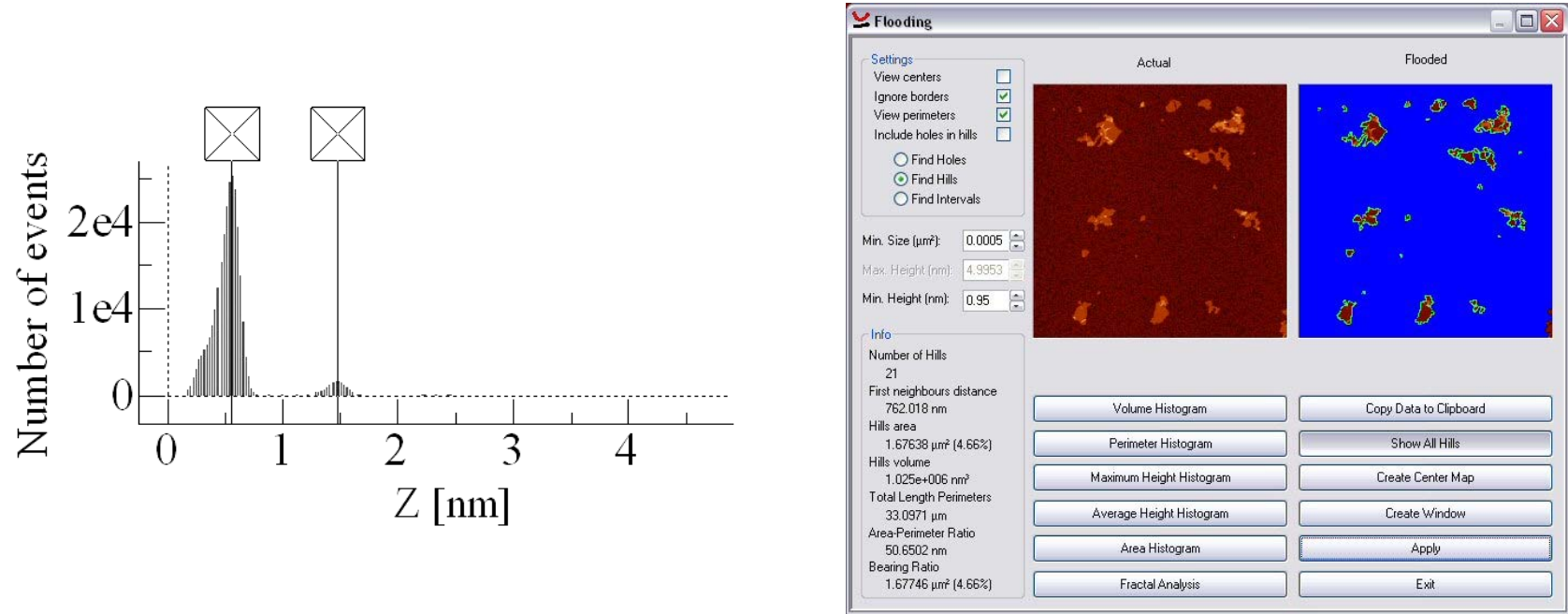

Figure 1. AFM characterization: a) Height histogram (left) and b) Flooding window for measuring the area of the sheets (right).

\section{Results and discussion}

The particle size of the graphites was kept as close as possible in order to avoid possible effects on the size of the GO sheets. For these reasons, the two parent cokes were ground to a specific particle size before graphitization in order to overcome the inherent grindability problems of graphite. Since cokes with a smaller size of optical texture (size, morphology and orientation of the crystalline structures) exhibit a greater mechanical strength than those of a larger size, it was not possible to obtain graphites with exactly the same particle size distribution as that initially intended, but the particle 
size did not exceed $80 \mu \mathrm{m}$, as confirmed experimentally. From the particle size distribution analysis (Table 1), the higher proportion of larger particles in the case of G2 (20\% of the particles in the range of $50-80 \mu \mathrm{m}$ as compared to $3.5 \%$ in the case of $\mathrm{G} 1$ ) is apparent. Graphites G1 and G2 have a carbon content of more than 99.9 wt \% and they are ash free. The optical texture [17], as determined by polarized light microscopy, confirms the homogeneous crystallinity of both graphites, which ranges from very well orientated structures known as flow domains (10-20 $\mu \mathrm{m}$ width, 50-100 length) for the more crystalline graphite (G1, Figure 2, top) to small mosaics $(1.5-5 \mu \mathrm{m})$ for the less crystalline one (G2, Figure 2, bottom). These results are supported by the Lc and La values obtained by XRD, which are 26.2 and $65.4 \mathrm{~nm}$ for G1 and 19.0 and $43.5 \mathrm{~nm}$ for G2, respectively.
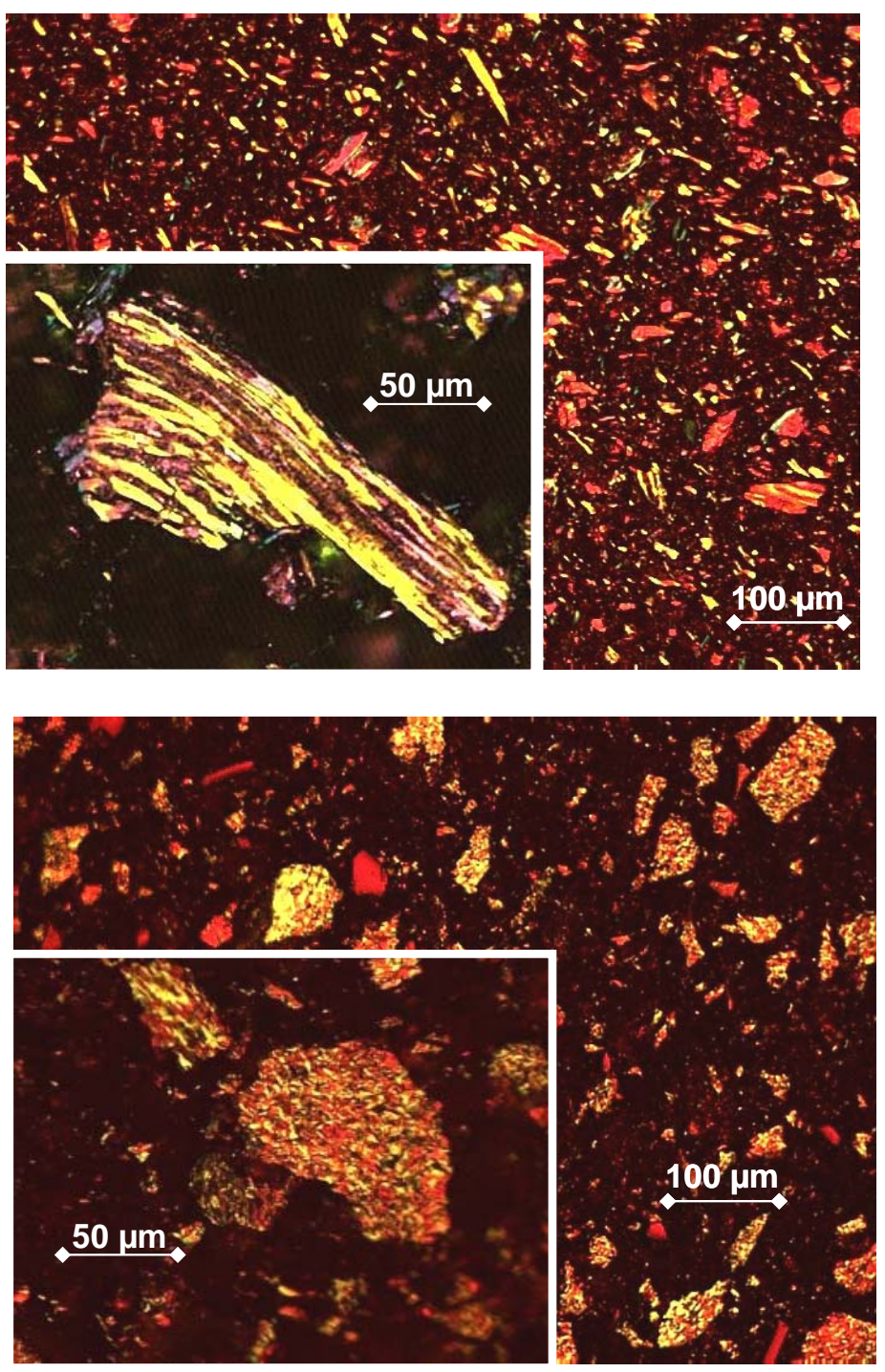
Figure 2. Optical micrographs of graphites G1 (top) and G2 (bottom) showing the differences in particle size and optical texture of flow domains (G1) and mosaics (G2).

Table 1 Characteristics of graphites.

\begin{tabular}{cccccccc}
\hline & \multirow{2}{*}{ C (\%) } & \multirow{2}{*}{ Ash (\%) } & \multicolumn{2}{c}{ XRD } & \multicolumn{3}{c}{ Particle diameter (\%) } \\
\cline { 3 - 7 } & & & Lc $(\mathrm{nm})$ & $\mathrm{La}(\mathrm{nm})$ & $2-20(\mu \mathrm{m})$ & $20-50(\mu \mathrm{m})$ & $50-80(\mu \mathrm{m})$ \\
\hline G1 & 99.93 & 0.02 & 26.2 & 65.4 & 71.8 & 23.7 & 3.5 \\
G2 & 99.91 & 0.01 & 19.0 & 43.5 & 38.7 & 38.6 & 20.0 \\
\hline
\end{tabular}

a. Elemental analysis, weight percent, b. TGA, weight percent.

The Raman spectra of the parent graphites (Figure 3) exhibit a characteristic strong G band at $1577 \mathrm{~cm}^{-1}$ for $\mathrm{G} 1$ and at $1580 \mathrm{~cm}^{-1}$ for $\mathrm{G} 2$ which are attributed to the vibration of $\mathrm{sp}^{2}$-bonded carbon atoms in a two-dimensional hexagonal lattice, as well as a weak D band at $1349 \mathrm{~cm}^{-1}$ for $\mathrm{G} 1$ and at $1338 \mathrm{~cm}^{-1}$ for $\mathrm{G} 2$ which is caused by the graphite edges or imperfections. Consequently, the intensity of $\mathrm{I}_{\mathrm{D}} / \mathrm{I}_{\mathrm{G}}$ in graphite $\mathrm{G} 2\left(\mathrm{I}_{\mathrm{D}} / \mathrm{I}_{\mathrm{G}} \mathrm{G} 2: 0.089\right.$ vs $\left.\mathrm{I}_{\mathrm{D}} / \mathrm{I}_{\mathrm{G}} \mathrm{G} 1: 0.045\right)$ is double that of $\mathrm{G} 1$.

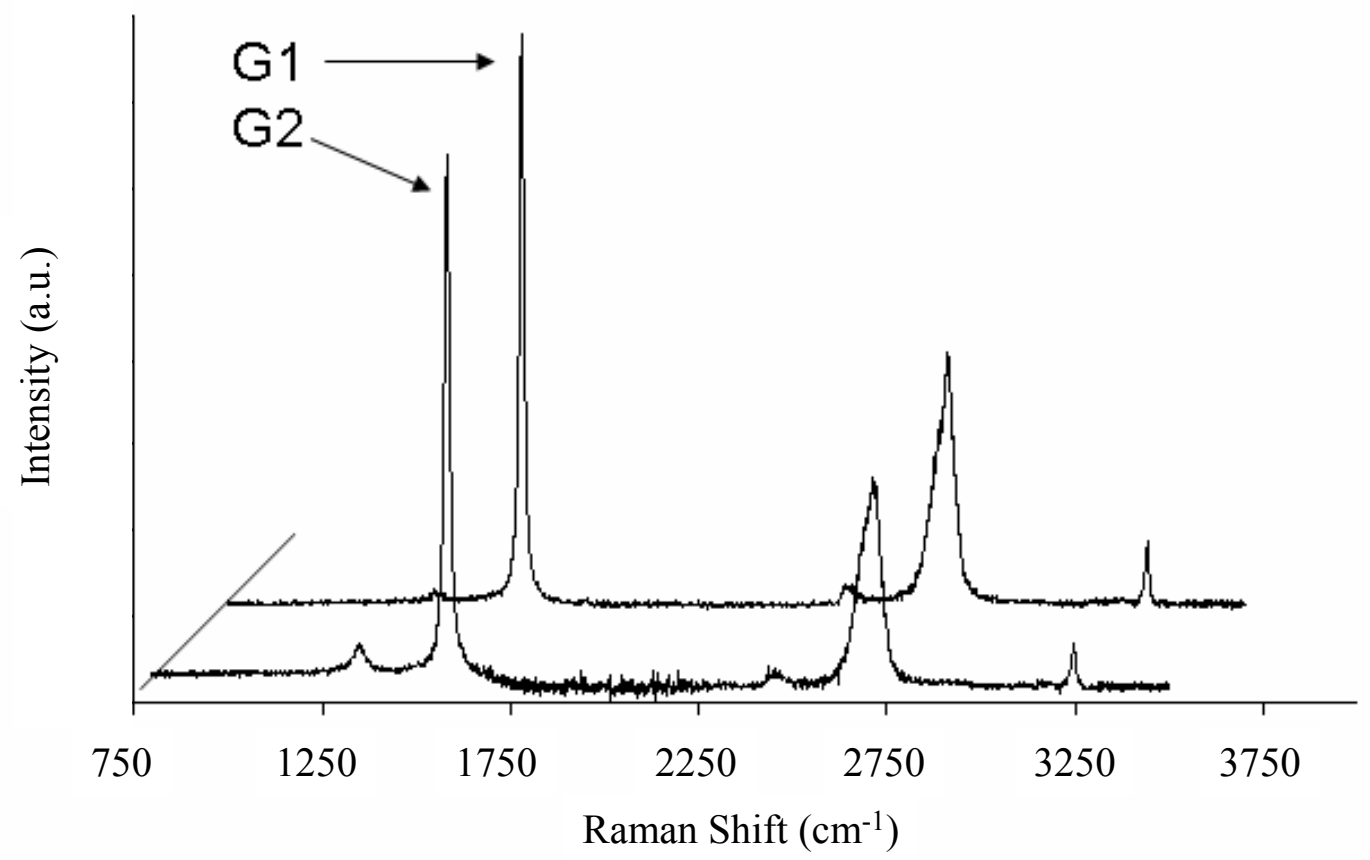

Figure 3. Raman spectra of graphites G1 and G2. 
The yield of the two GOs was always higher than $80 \mathrm{wt}$. \%. Their dispersive properties are known to be critically dependent on the nature and concentration of the functionalities at the GO edges [18]. The suspension of the two GOs in water (highly polar) exhibited a high stability at a concentration of $1 \mathrm{mg} \mathrm{mL}^{-1}$ for at least 6 months. However, clear differences became apparent when analyzing the stability of the suspensions obtained in N-methyl-2-pyrrolidinone (NMP, medium polarity) and tetrahydrofurane (THF, low polarity). Whereas the suspensions of GO obtained from the more crystalline graphite (GO1) remained partially stable in these solvents for at least 1 month (Figure 4), those obtained from the less crystalline graphite (GO2) exhibited a very low stability in NMP and showed no stability at all in THF. This behavior suggests that the oxygen functionalities and/or their distribution make GO2 more polar, on average, than GO1, probably as a consequence of the larger proportion of hydroxyl and carboxyl surface groups, as shown below.

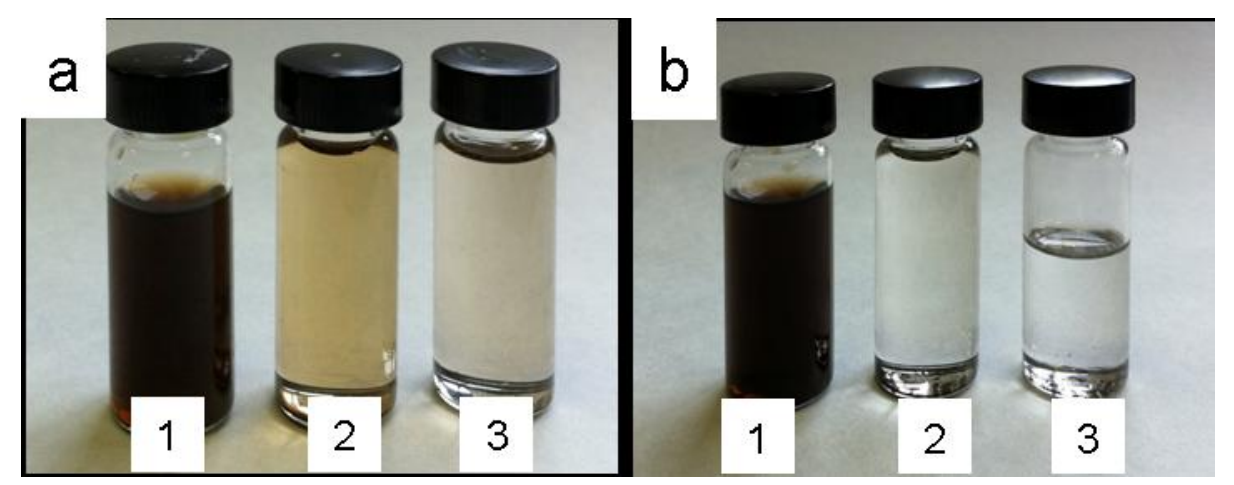

Figure 4. Images of suspensions of $\mathrm{GO} 1$ (a) and $\mathrm{GO} 2$ (b) in water (1), thetrahydrofurane (2) and N-Methyl-2 pyrrolidinone (3) at a concentration $1 \mathrm{mg} \mathrm{mL}^{-1}$ after 6 months. 
The Raman spectra of GO1 and GO2 exhibit a typical graphene-like pattern (Figure 5). What is of interest in this case is that the $\mathrm{I}_{\mathrm{D}} / \mathrm{I}_{\mathrm{G}}$ ratio of GO1 $(0.89)$ is slightly lower than for $\mathrm{GO} 2$ (0.92), indicating that the GO2 sheets have more defects in their structure.

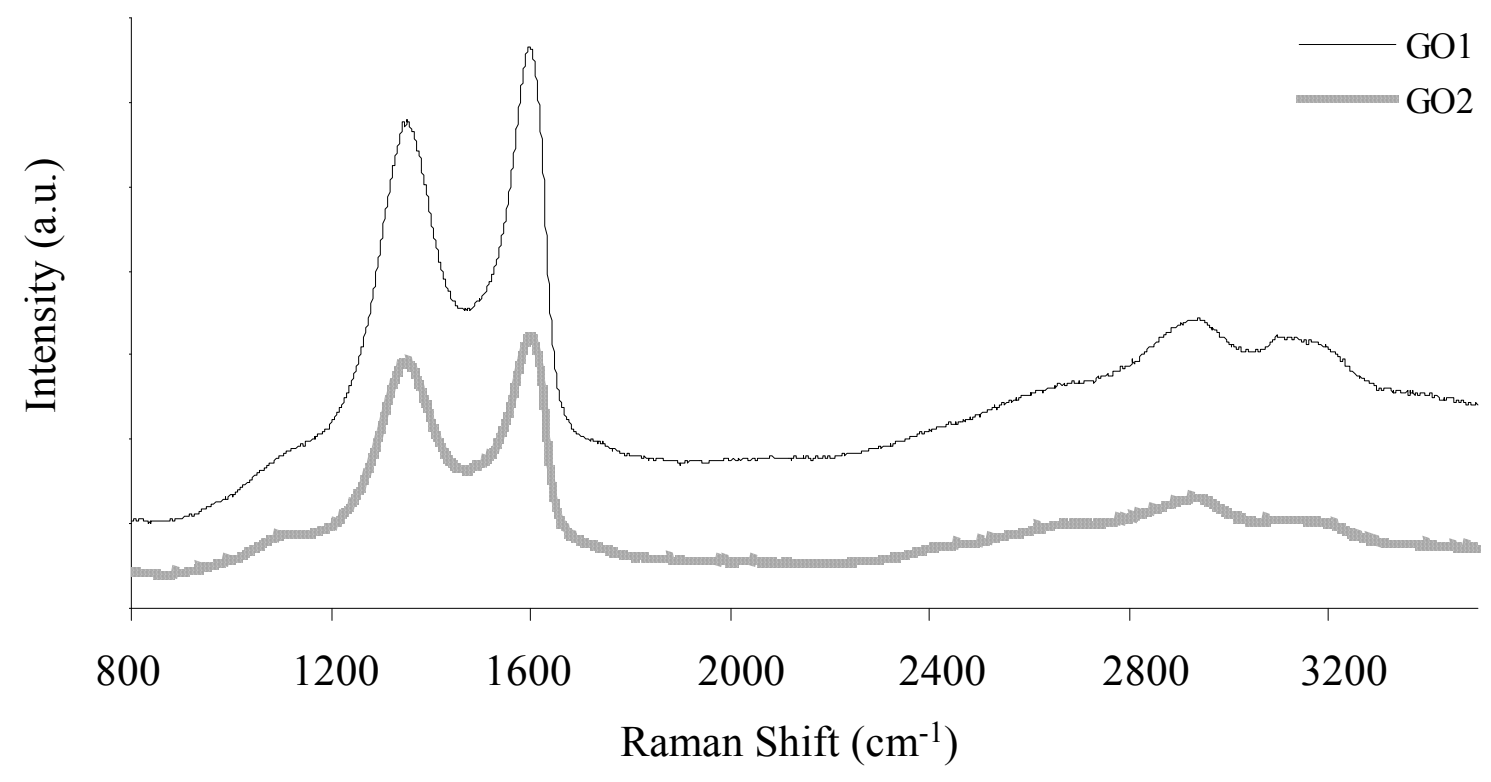

Figure 5. Raman spectra of graphene oxides GO1 and GO2.

The atomic concentrations of the graphene oxides $\mathrm{GO} 1$ and $\mathrm{GO} 2$ were evaluated by means of XPS (Figure 6). C/O ratios of $\sim 2.0$ were obtained for both samples. However, the oxide from the graphite with the larger crystal size (GO1) exhibits an increased amount of $\mathrm{sp}^{2} \mathrm{C}-\mathrm{C}$ bonds (45.12 for GO1 and $36.18 \%$ for GO2), as well as a higher amount of $\mathrm{C}-\mathrm{O}-\mathrm{C}$ bonds that are probably located at the basal planes of the GO layer (39.40 for GO1 and $13.76 \%$ for GO2). In contrast, GO2 exhibits a larger amount of $\mathrm{sp}^{3}$ $\mathrm{C}$ atoms (the $\mathrm{sp}^{3} \mathrm{C}-\mathrm{C}$ bonds representing 11.30 for $\mathrm{GO} 2$ and $4.93 \%$ for GO1), also corresponding to hydroxyl (1.86 for GO1 and $20.28 \%$ for GO2) and carboxyl groups 
located at the edges of the sheets (10.71 for GO1 and $19.47 \%$ for GO2). This explains the lower stability of GO2 in NMP and THF described above. The XPS results are corroborated by FTIR (Figure 7). The more pronounced weight loss in GO2 at lower temperatures (up to $200{ }^{\circ} \mathrm{C}$ ) observed by TGA is a further evidence of the presence of more unstable oxygen functional groups; carboxyl and hydroxyl groups (Figure 8) [19].

a)

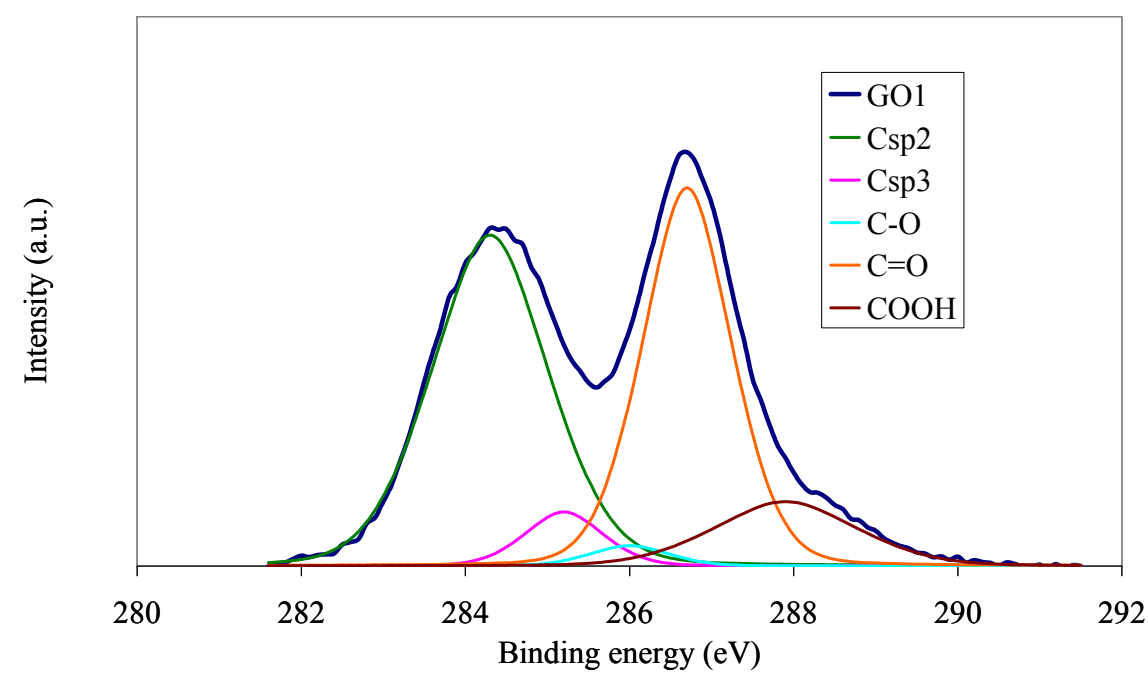

b)

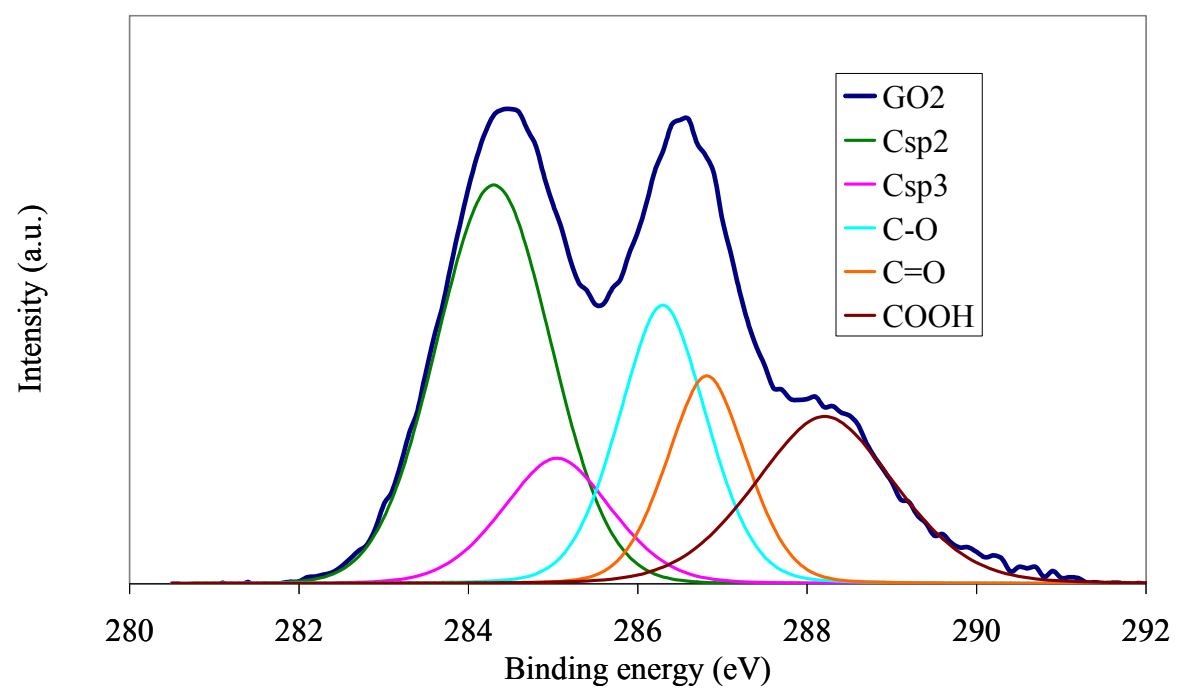

Figure 6. XPS curve fitting of $\mathrm{C} 1$ s spectra of graphene oxides $\mathrm{GO} 1$ (a) and GO2 (b). 


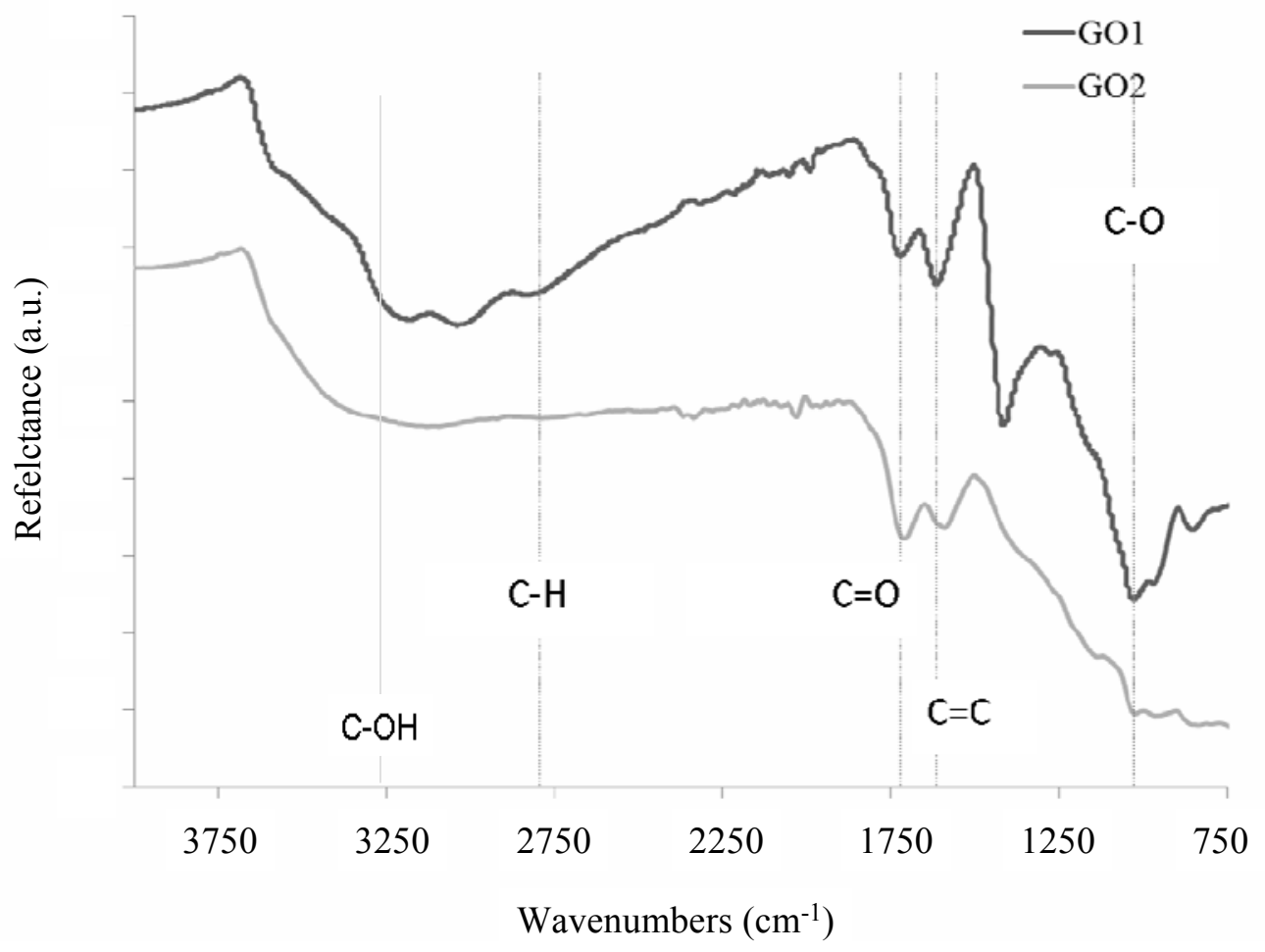

Figure 7. FTIR spectra of graphene oxides GO1 and GO2.

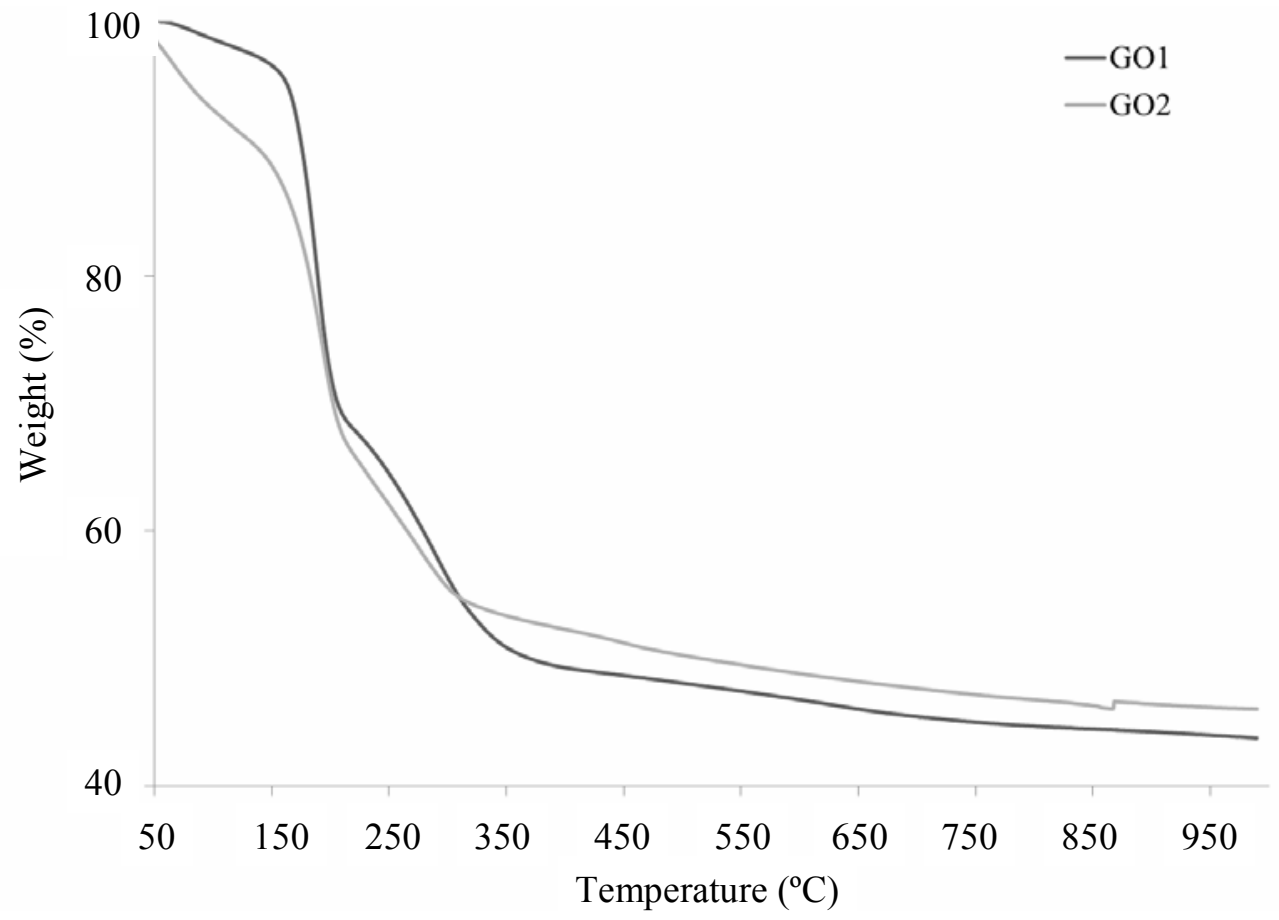

Figure 8. TGA curves of graphene oxides GO1 and GO2. 
The size and height of the GOs as measured by means of AFM imaging and profiling, show a dominant presence of "monolayers" in the two oxides, with an average height of $0.92 \pm 0.10 \mathrm{~nm}$ for $\mathrm{GO} 1$ and $0.90 \pm 0.12 \mathrm{~nm}$ for GO2 [20-22] (Figure 9). TEM corroborated the presence of single folded sheets and no substantial differences between the two GOs could be observed (Figure 10). The most important finding obtained by AFM was that the average area of the GOs sheets was a function of the crystal structure of the parent graphite. The average area $\left(\mu \mathrm{m}^{2} \times 10^{-3}\right)$ ranged from $57 \pm 3$ for GO1 (prepared from the graphite with the larger crystal size) to $3 \pm 1$ for GO2 (obtained from the graphite with the smaller crystal size). This effect is even more evident if one takes into account that G2 exhibits a slightly larger particle size than G1. As both oxides have similar oxygen content and they were sonicated during the same period of time, these results clearly show that the size of the GO sheets obtained, is more a function of the crystal size of the parent graphite than of its actual particle size.
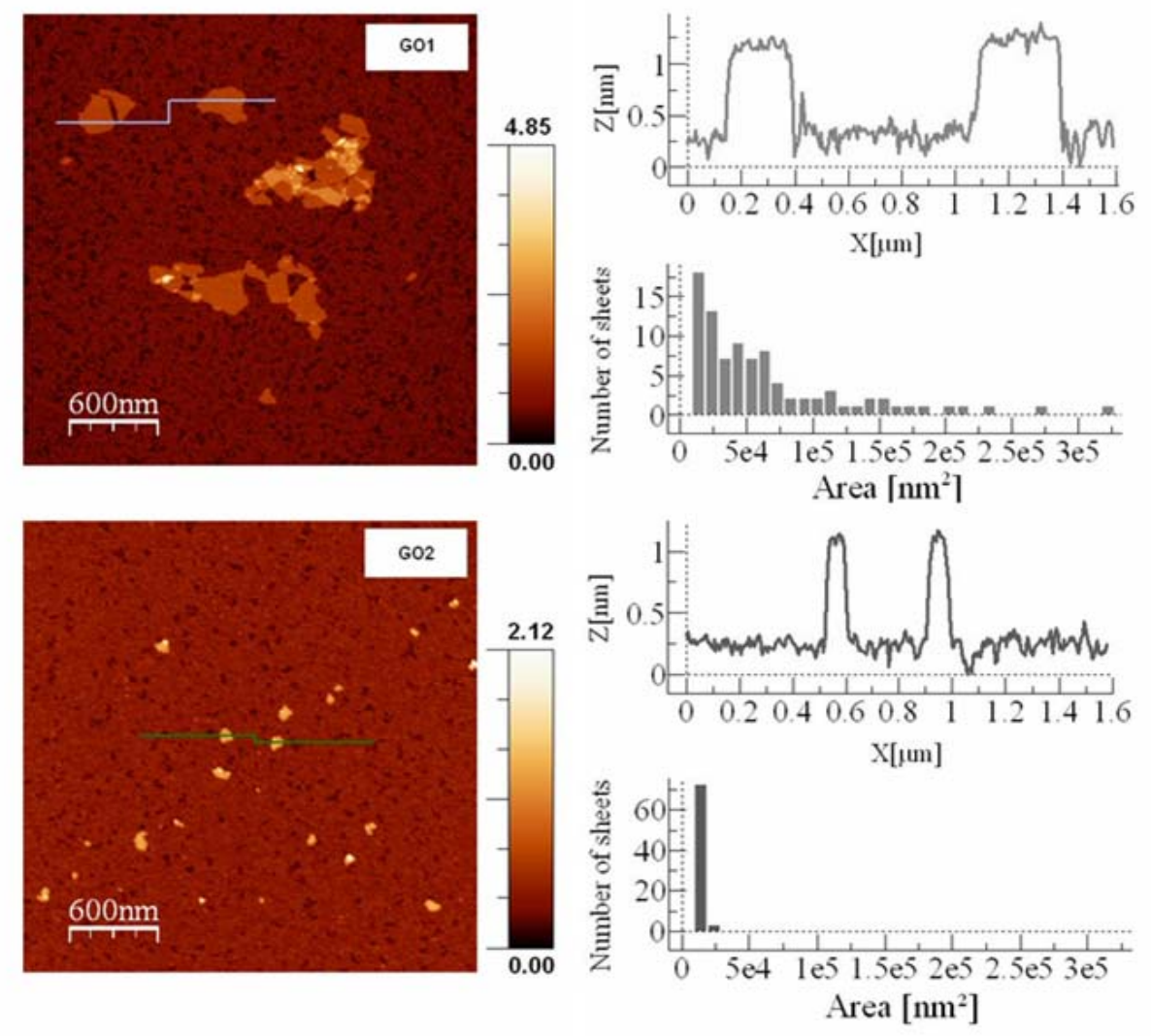
Figure 9. AFM Images of GO sheets. The sheets are $1 \mathrm{~nm}$ thick. The horizontal lines indicate the sections corresponding to the traces shown on the right. The histograms show the area distributions of the total amount of GO sheets analyzed.

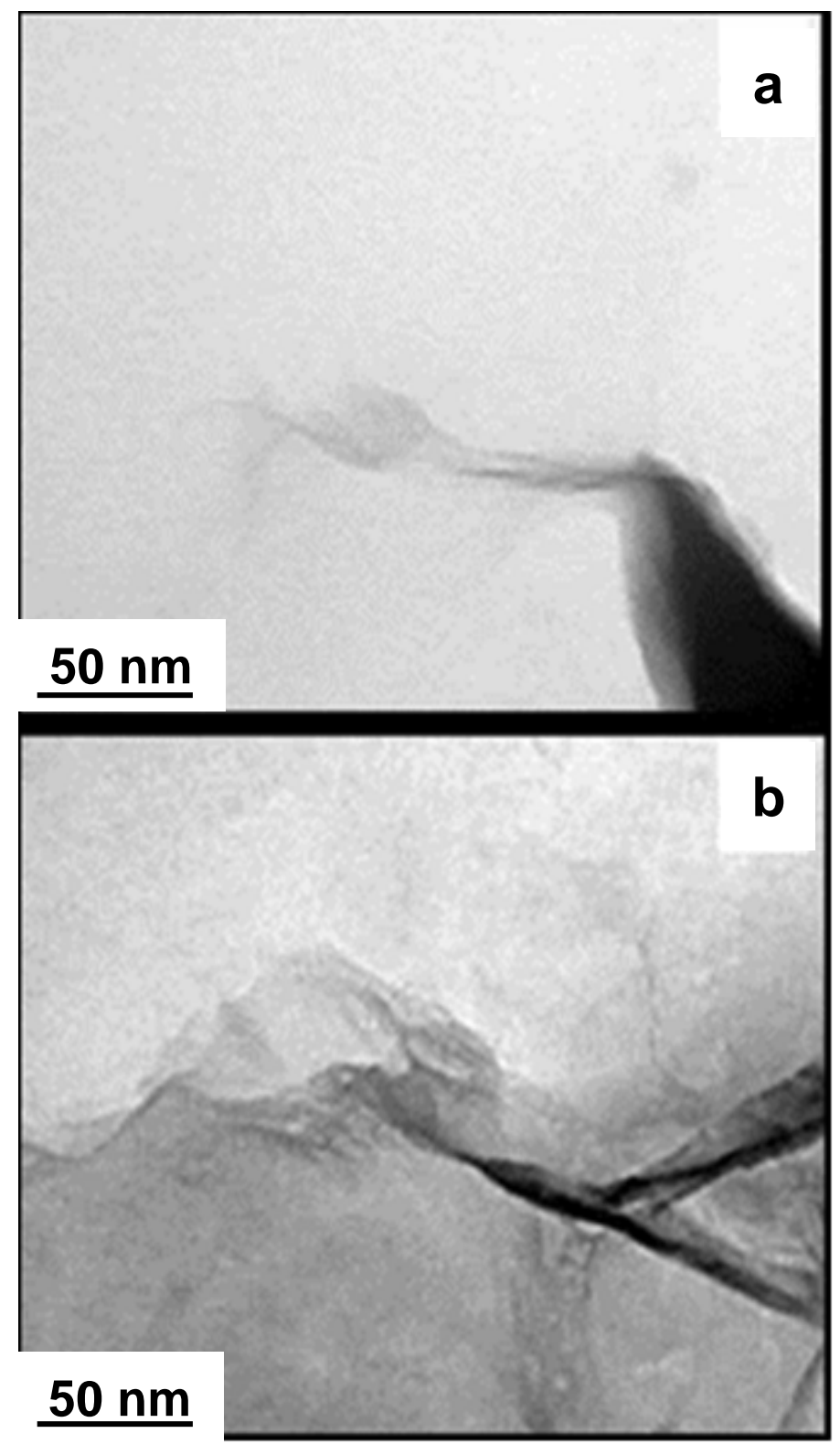

Figure 10. TEM images showing individual platelets extending from a particle composed of agglomerated GO sheets of (a) GO1 and (b) GO2.

Our findings suggest a possible mechanism for the attack of oxygen on graphite. In graphites with a smaller crystal size (optical texture made up of mosaics) the attack by 
oxygen occurs predominantly at the boundaries of the crystals, yielding GO sheets of a smaller size and with more abundant hydroxyl and carboxyl groups, which are mainly located at the edges. In more ordered graphites (optical texture made up of flow domains) oxidation occurs through the formation of oxygen functionalities mainly at the basal planes, yielding larger size sheets with a predominance of epoxy groups (Figure $11)$.

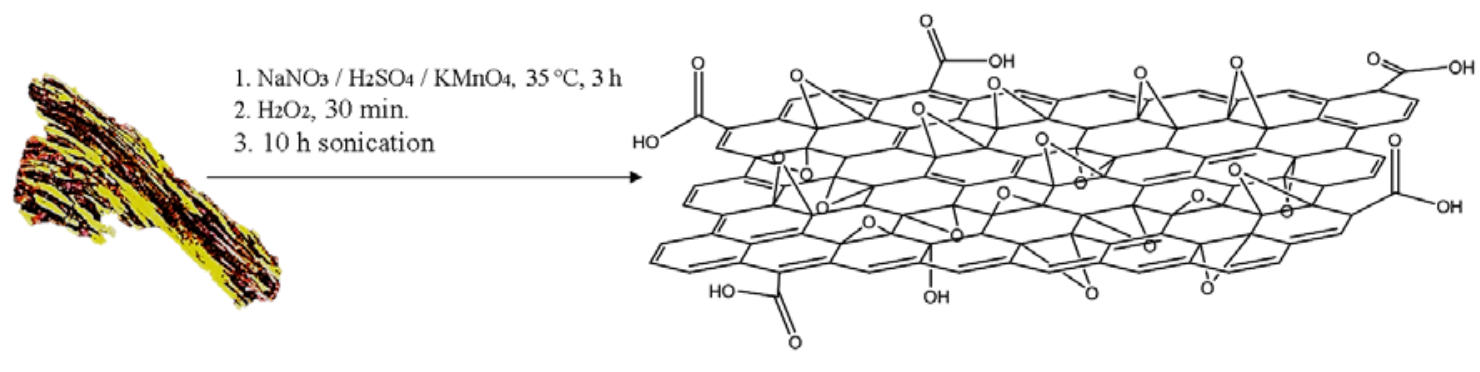

G1

GO1

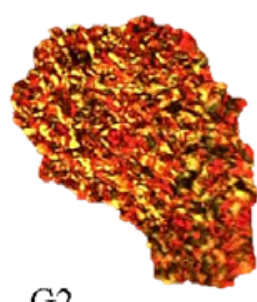

1. $\mathrm{NaNO}_{3} / \mathrm{H}_{2} \mathrm{SO}_{4} / \mathrm{KMnO}_{4}, 35^{\circ} \mathrm{C}, 3 \mathrm{~h}$

2. $\mathrm{H}_{2} \mathrm{O}_{2}, 30 \mathrm{~min}$.

3. $10 \mathrm{~h}$ sonication

G2

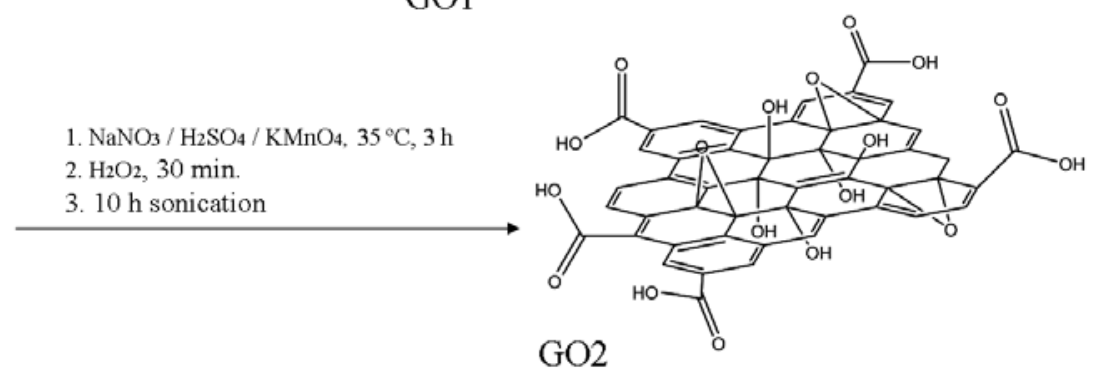

Figure 11. Schematic model of a sheet of (top) GO1 and (bottom) GO2 with their respective parent graphites.

\section{Conclusions}

Graphites of different crystalline structure yielded monolayer GOs with an average area varying from $57.0 \times 10^{-3} \mu \mathrm{m}^{2}$ (in the case of the more crystalline graphite) to $3.0 \times 10^{-3}$ $\mu \mathrm{m}^{2}$ (in the case of the less crystalline graphite). Significantly, the oxide obtained from the less crystalline graphite was found to contain a larger proportion of hydroxyl and carboxyl groups, whereas the oxide from the more crystalline graphite contained 
predominantly epoxy groups, reflecting their different chemical behavior. These results provide direct evidences of the active role of the crystal boundaries of each graphite layer in the oxidation process and suggest that it may be possible to tailor the size and chemistry of GOs from the characteristics of the parent graphite.

ACKNOWLEDGMENT. The authors thank MICINN (CONSOLIDER INGENIO 2010, Ref. CSD2009-00050) for their financial support. Dr. Patricia Alvarez thanks MICINN for her Ramon y Cajal contract. Cristina Botas acknowledges a fellowship from FICYT (Gobierno del Principado de Asturias). Special thanks go to Prof. Julio Gómez Herrero, Dr. Cristina Gómez-Navarro and Dr. Adriana Gil for their valuable suggestions.

\section{REFERENCES}

[1] Rao CNR, Sood AK, Subrahmanyam KS, Govindaraj A. Graphene: the new twodimensional nanomaterial. Angew. Chem.2009; 48: 7752 - 77

[2] Park S, Ruoff RS. Chemical methods for the production of graphenes. Nat. Nanotechnol 2010; 217-24.

[3] Jiang H. Chemical Preparation of Graphene-Based Nanomaterials and Their Applications in Chemical and Biological Sensors. Small. DOI: 0.1002/smll.201002352 (2011).

[4]Huang X, Qi XY, Boey F, Zhang H. Graphene-Based Composites. Chem. Soc. Rev. DOI: 10.1039/C1CS15078B (2011). 
[5] Huang X, Yin Z, Wu1 S, Qi X, He1 Q, Zhang Q, Yan Q, Boey F, Zhang H.

Graphene-Based Materials: Synthesis, Characterization, Properties, and Applications. Small, DOI: 10.1002/smll.201002009 (2011)

[6] Avouris P. Graphene: electronic and photonic properties and devices. Nano Lett. 2012; 10: 4285-94.

[7] Bagri A, Mattevi C, Acik M, Chabal Y, Chhowalla M, Shenoy V. Structural evolution during the reduction of chemically derived graphene oxide. Nature Chem. $2010 ; 2 ; 581-7$.

[8] Wu Z, Ren W, Gao L, Liu B, Jiang C, Cheng H. Synthesis of high-quality graphene with a pre-determined number of layers. Carbon 2009; 47 (2): 493-9.

[9] Inagaki M, Kim YA, Endo M. Graphene: preparation and structural perfection. J. Mat. Chem. 2011; 21: 3280-94.

[10] Yoshida A, Hishiyama Y. Electron channeling effect on highly oriented graphites-size evaluation and oriented mapping of crystals. J. Mat. Res. 1990; 7: $1400-5$.

[11] Evans EL, López-González JdeD, Martin-Rodríguez A, Rodríguez-Reinoso F. Kinetics of the formation of graphite oxide. Carbon 1975; 13 (6): 461-4.

[12] Alvarez P, Granda M, Sutil J, Menéndez R, Fernández JJ, Viña JA, et al. Characterization and pyrolysis behavior of novel anthracene oil derivatives. Ener. Fuel 2008; 22: 4077-86.

[13] Hummers WS, Offeman RE. Preparation of Graphitic Oxide. J. Am. Chem. Soc. 1958; 80: 1339.

[14] Sherwood PMA. Auger and X-ray Photoelectron Spectroscopy. In: Briggs D and Seah MP editors. Practical Surface Analysis, vol. 1, New York; Wiley; 1990 p.574. 
[15] Yang D, Velamakanni A, Bozoklu G, Park S, Stoller M, Richard D, et al. Chemical analysis of graphene oxide films after heat and chemical treatments by Xray photoelectron and Micro-Raman spectroscopy. Carbon 2009; 47 (20): 145-52.

[16] Shafiei M, Spizzirri PG, Arsat R, Yu J, du Plessis J, Dubin S, et al. Platinum/graphene nanosheet/SiC contacts and their application for hydrogen gas sensing. J. Phys. Chem. C 2010; 114: 13796-801.

[17] Forrest RA, Marsh H. Reflection interference colours in optical microscopy of carbon. Carbon 1977; 15: 348-9.

[18] Li D, Muller MB, Gilje S, Kaner RB, Wallace GG. Processable aqueous dispersions of graphene nanosheets. Nature Nanotechnology 2008; 3: 101-5.

[19] Figueiredo JL, Pereira MFR,. Freitas MMA, Orfao JJM. Modification of the surface chemistry of activated carbons. Carbon 1999; 37: 1379-1389.

[20] Stankovich S, Piner RD, Chen XQ, Wu NQ, Nguyen ST, Ruoff RS. Stable aqueous dispersions of graphitic nanoplatelets via the reduction of exfoliated graphite oxide in the presence of poly(sodium 4-styrenesulfonate). J. Mater. Chem. 2006; 16 : $155-8$.

[21] Schniepp HC, Li JL, McAllister MJ, Sai H, Herrera-Alonso M, Adamson DH, et al. Functionalized single graphene sheets derived from splitting graphite oxide. J. Phys. Chem. B 2006; 110: 8535-39.

[22] Gomez-Navarro C, Weitz RT, Bittner AM, Scolari M, Mews A, Burghard M, et al. Electronic transport properties of individual chemically reduced graphene oxide sheets. Nano Lett. 2007; 7: 3499-503. 


\section{Table captions}

Table 1 Characteristics of graphites

\section{Figure captions}

Figure 1. AFM characterization: a) Height histogram (left) and b) Flooding window for measuring the area of the sheets (right).

Figure 2. Optical micrographs of graphites G1 (top) and G2 (bottom) showing the differences in particle size and optical texture of flow domains (G1) and mosaics (G2).

Figure 3. Raman spectra of graphites G1 and G2.

Figure 4. Images of suspensions of GO1 (a) and $\mathrm{GO} 2$ (b) in water (1), thetrahydrofurane (2) and N-Methyl-2 pyrrolidinone (3) at a concentration $1 \mathrm{mg} \mathrm{mL}^{-1}$ after 6 months.

Figure 5. Raman spectra of graphene oxides GO1 (a) and GO2 (b).

Figure 6. XPS curve fitting of $\mathrm{C} 1 \mathrm{~s}$ spectra of graphene oxides GO1 (a) and GO2 (b).

Figure 7. FTIR spectra of graphene oxides GO1 and GO2.

Figure 6. XPS curve fitting of C1s spectra of graphene oxides GO1 (a) and GO2 (b).

Figure 8. TGA curves of graphene oxides GO1 and GO2. 
Figure 9. AFM Images of GO sheets. The sheets are $1 \mathrm{~nm}$ thick. The horizontal lines indicate the sections corresponding to the traces shown on the right. The histograms show the area distributions of the total amount of GO sheets analyzed.

Figure 10. TEM images showing individual platelets extending from a particle composed of agglomerated GO sheets of (a) GO1 and (b) GO2.

Figure 11. Schematic model of a sheet of (top) GO1 and (bottom) GO2 with their respective parent graphites. 